\title{
ON STURMIAN THEORY FOR SECOND ORDER SYSTEMS
}

\author{
SHAIR AHMAD
}

ABSTRACT. A generalization of the Sturm Comparison Theorem is given to nonselfadjoint second order linear systems. In addition, a theorem, involving the existence of a solution with strictly positive components, is proven. Counterexamples are given to show that the theorems are false without the stated assumptions.

Introduction. Consider the vector differential equations

$$
x^{\prime \prime}+A(t) x=0
$$

and

$$
y^{\prime \prime}+B(t) y=0
$$

where $A(t)=\left(a_{i j}(t)\right)$ and $B(t)=\left(b_{i j}(t)\right)$ are continuous $n \times n$ matrices. A number $\beta$ is a conjugate point of $\alpha, \alpha<\beta$, relative to (1) if (1) has a nontrivial solution vanishing at $\alpha$ and $\beta$. If no nontrivial solution of (1) vanishes twice in an interval $I$, then (1) is disconjugate on $I$. Paralleling the work of Morse [9] for selfadjoint systems, in [2] we gave a generalization of the Sturm Comparison Theorem for nonselfadjoint systems. In particular, we showed that if $b_{i j}(t) \geqslant a_{1 j}(t) \geqslant 0,1 \leqslant i$, $j \leqslant n$, with $b_{i j}(\bar{t})>a_{i j}(\bar{t})$ at some point $\bar{t}, \bar{t} \in[\alpha, \beta]$ (where $\beta$ is the first conjugate point of $\alpha$ relative to (1)), then the first conjugate point of $\alpha$ relative to (2) is less than the first conjugate point of $\alpha$ relative to (1). Later, in [4] it was shown that with the additional assumption that $A(t)$ and $B(t)$ be symmetric, the strict inequality $b_{i j}(\bar{t})>a_{i j}(\bar{t})$ can be relaxed to hold only along the diagonal elements, i.e. $b_{1 i}(\bar{t})>$ $a_{i i}(t)$. The purpose of our first theorem is to show that this result holds without the symmetry assumption on $A(t)$ and $B(t)$. Furthermore, we give a simple example to show that not only the comparison theorem established here but also those established in [ 2 and 4 ] are all false without the nonnegativity assumption on the elements of the coefficient matrices. This should eliminate any doubt about the importance of the nonnegativity assumption made in the earlier works [1-6].

In [1] it was shown that if (1) is disconjugate on $[a, \infty)$ and if $A(t)$ is a symmetric matrix with nonnegative elements, which is irreducible at some point in $[a, \infty)$, then (1) has a solution which vanishes at $a$ and all of whose components are strictly positive on $(a, \infty)$. This result was later established [6] without the symmetry condition. In both proofs the existence of a nontrivial solution with nonnegative components followed independent of the irreducibility condition. In this paper, we

Received by the editors June 21, 1982.

1980 Mathematics Subject Classification. Primary 34A25: Secondary 34C10.

Key words and phrases. Conjugate point, disconjugate, irreducible, symmetric, selfadjoint, solution. 
give an example, which is somewhat surprising, to show that a solution with strictly positive components may not exist without the irreducibility assumption. However, our second theorem shows that in the selfadjoint case such a solution does exist without the irreducibility condition on the coefficient matrix.

As in [4], the proofs of both of our theorems make use of induction on the order of the coefficient matrices. For the convenience of the reader, we point out that several of the results which were proven earlier and are used here have been stated in a unified manner in [4].

\section{Theorems and examples.}

THEOREM 1. Assume that $\alpha$ and $\beta$ are the first conjugate points of a relative to (1) and (2), respectively. Suppose that $b_{i j}(t) \geqslant a_{i j}(t) \geqslant 0,1 \leqslant i, j \leqslant n$, with $b_{i i}(\bar{t})>a_{i i}(\bar{t})$ for some $i \in(a, \alpha)$. Then $\beta<\alpha$.

Proof. Since $b_{i j}(t) \geqslant a_{i j}(t) \geqslant 0$, it follows (see [10]) that $\beta \leqslant \alpha$. We shall show that it is impossible to have $\beta=\alpha$. Assume, on the contrary, that $\beta=\alpha$. There exists (see [2]) a nontrivial solution $u(t)=\operatorname{col}\left(u_{1}, \ldots, u_{n}\right)$ of (1) with $u_{i}(t) \geqslant 0,1 \leqslant i \leqslant n$. for $t \in(a, \alpha)$ such that $u(a)=u(\alpha)=0$. We consider two cases.

Case 1. Assume that $u_{i}(t)>0$ for all $t, t \in(a, b)$, and for all $i, i=1, \ldots, n$. There exists a nontrivial solution $w(t)=\operatorname{col}\left(w_{1}, \ldots, w_{n}\right)$ such that

$$
w^{\prime \prime}+B^{T}(t) w=0, \quad w(a)=w(\alpha)=0,
$$

and $w_{i}(t) \geqslant 0,1 \leqslant i \leqslant n$, for all $t$ in $(a, \alpha)$, where $B^{T}$ denotes the transpose of the matrix $B$. This follows from [2] and the fact (see [5]) that $\alpha=\beta$ is also the first conjugate point of $a$ relative to (3). Now, multiplying $u^{\prime \prime}+A(t) u=0$ by $w^{T}(t)$ and (3) by $-u^{T}(t)$, and adding, we have

$$
w^{T} u^{\prime \prime}-u^{T} w^{\prime \prime}=u^{T} B^{T} w^{\prime}-w^{T} A u .
$$

Therefore, since $u^{T} B^{T} w=w^{T} B u$,

$$
\left(w^{T} u^{\prime}-u^{T} w^{\prime}\right)^{\prime}=w^{T}(B-A) u .
$$

Integrating from $a$ to $\alpha$, we obtain

$$
\int_{a}^{\alpha} w^{T}(s)(B(s)-A(s)) u(s) d s=0 .
$$

which is a contradiction, since it is easy to verify that $b_{i i}(\bar{t})-a_{i i}(\bar{t})>0, w_{i}(s) \geqslant 0$. and $u_{i}(s)>0,1 \leqslant i \leqslant n$, imply

$$
\int_{a}^{\alpha} w^{T}(s)(B(s)-A(s)) u(s) d s>0 .
$$

Case 2. Assume that at least one component of $u(t)$ vanishes on $(a, \alpha)$. By Lemma 1 of [1], if a component $u_{i}(t)$ of $u(t)$ vanishes at one point of $(a, \alpha)$ then $u_{i}(t) \equiv 0$ on $[a, \alpha]$. By renumbering, if necessary, the components of $u(t)$ and the $n$ equations of the system in (1), we may assume that $u_{j}(t)>0$ for $j=1, \ldots, k$, and $u_{j}(t) \equiv 0$ for $j=k+1, \ldots, n$. As in [7], we partition $A(t)$ as

$$
A(t)=\left[\begin{array}{ll}
M(t) & N(t) \\
O(t) & P(t)
\end{array}\right],
$$


where $M$ is $k \times k, O$ is $(N-k) \times k, N$ is $k \times(n-k)$, and $N$ is $(n-k) \times(n-k)$. Let us write $u(t)$ as

$$
u(t)=\left[\begin{array}{c}
\hat{u}(t) \\
\hat{0}
\end{array}\right]
$$

where $\hat{u}(t)$ consists of the first $k$ components of $u(t)$ and $\hat{0}$ consists of $n-k$ zero elements. We note that

$$
\hat{u}^{\prime \prime}+M(t) \hat{u}=0
$$

and that all the elements of $O(t)$ are zero. Clearly, $\alpha$ is a conjugate point of $a$ relative to (4). We note that the conjugate points of $a$ relative to (4) are the same as those relative to

$$
x^{\prime \prime}+C(t) x=0
$$

where

$$
C(t)=\left[\begin{array}{ll}
M(t) & 0 \\
0 & 0
\end{array}\right] .
$$

By the comparison theorem in [10], if $\gamma$ is the first conjugate point of $a$ relative to (5) then $\gamma \geqslant \alpha$. On the other hand, $\alpha$ is a conjugate point of $a$ relative to (5). Therefore, $\alpha$ must be the first conjugate point of $a$ relative to (5) and, hence, relative to (4). Now, we partition the matrix $B$ as

$$
B=\left[\begin{array}{ll}
\hat{M}(t) & \hat{N}(t) \\
\hat{O}(t) & \hat{P}(t)
\end{array}\right],
$$

where $\hat{M}(t)$ is $k \times k$, etc. By the induction hypothesis, if $\hat{\gamma}$ is the first conjugate point of $a$ relative to $y^{\prime \prime}+\hat{M}(t) u=0$, then $\hat{\gamma}<\alpha$. Using an argument similar to the one given above and using the intermediary matrix

$$
\hat{C}(t)=\left[\begin{array}{ll}
\hat{M}(t) & 0 \\
0 & 0
\end{array}\right],
$$

it follows that the first conjugate point $\beta$ of $a$ relative to (2) satisfies the inequality $\beta \leqslant \hat{\gamma}<\alpha$; contradicting $\beta=\alpha$. This completes the proof.

The following example shows that not only the preceding theorem, but also the comparison theorems established earlier (see [ 2 and 4]) For the selfadjoint and nonselfadjoint systems are all false without the nonnegativity assumption.

EXAMPLE 1. Let $A(t)$ and $B(t)$ in (1) and (2) be given by

$$
A(t)=\left[\begin{array}{ll}
0 & -4 \\
-4 & 0
\end{array}\right] \text { and } B(t)=\left[\begin{array}{ll}
1 & 0 \\
0 & 1
\end{array}\right]
$$

Then the first conjugate point of 0 relative to (2) is $\pi$. On the other hand, $y(t)=\operatorname{col}(\sin 2 t,-\sin 2 t)$ is a solution of the system

$$
y_{1}^{\prime \prime}-4 y_{2}=0, \quad y_{2}^{\prime \prime}-4 y_{1}=0
$$

and hence of (2). Therefore, $\pi / 2$ is a conjugate point of 0 relative to (1). 
THEOREM 2. Assume that $A(t)=\left(a_{i j}(t)\right)$ is an $n \times n$ continuous symmetric matrix with $a_{i j}(t) \geqslant 0$ for all $t$ in $[a, \infty)$. If

$$
x^{\prime \prime}+A(t) x=0
$$

is disconjugate on $[a, \infty)$, then it has a solution $u(t)=\operatorname{col}\left(u_{1}(t), \ldots, u_{n}(t)\right)$ such that $u(a)=0$ and $u_{i}(t)>0$ for $i=1, \ldots, n$, and $t \in(a, \infty)$.

Proof. Our proof is by induction on the family of $n \times n$ continuous symmetric matrices with nonnegative elements on $[a, \infty)$. The assertion of the theorem is obviously true for $n=1$. Assume that it holds for all such $k \times k$ matrices, $1 \leqslant k<n$. We want to show that it holds for the $n \times n$ matrix $A(t)$ in (1). There exists (see Theorem 4 of [1], or Theorem 3.2 of [6]) a nontrivial solution $u(t)=\operatorname{col}\left(u_{1}, \ldots, u_{n}\right)$ of (1) such that $u(a)=0$ and $u_{i}(t) \geqslant 0, i=1, \ldots, n ; t \geqslant a$. Assume that some component $u_{j}(t)$ of $u(t)$ vanishes at some point in $(a, \infty)$. Then, by Lemma 1 of [1], $u_{j}(t) \equiv 0$ on $[a, \infty)$. Let $D$ be a matrix obtained by rearranging the columns of the $n \times n$ identity matrix such that the first, say, $k$ components of $D u(t)$ are strictly positive on $(a, \infty)$, while the remaining $n-k$ components are identically zero. We note that the permutation matrix $D$ has the property that $D^{-1}=D^{T}$. Thus, we have

$$
z^{\prime \prime}+B(t) z=0
$$

where $B(t)=D A D^{T}$ and $z=D u$. Clearly, $B(t)$ is a symmetric matrix with nonnegative elements. Let $z(t)=\operatorname{col}(\phi(t), 0)$, where $\phi(t)$ consists of the positive components of $z(t)$. Hence, substituting in (6), we may write (6) as

$$
\left(\begin{array}{c}
\phi^{\prime \prime} \\
0
\end{array}\right)+\left(\begin{array}{ll}
R & S \\
S^{T} & U
\end{array}\right)\left(\begin{array}{l}
\phi \\
0
\end{array}\right)=0
$$

Since $0+S^{T} \cdot \phi+U \cdot 0=0$, it follows that $S^{T}=0=S$. Thus, we may rewrite the preceding system as

$$
\left(\begin{array}{c}
\phi^{\prime \prime} \\
0
\end{array}\right)+\left(\begin{array}{ll}
R & 0 \\
0 & U
\end{array}\right)\left(\begin{array}{l}
\phi \\
0
\end{array}\right)=0
$$

where $R$ and $U$ are symmetric. It is easy to see that

$$
x^{\prime \prime}+B(t) x=0
$$

is disconjugate on $[a, \infty)$. Using the comparison theorem in [10] and the intermediary matrix $\left(\begin{array}{ll}0 & 0 \\ 0 & U\end{array}\right)$ it follows that $v^{\prime \prime}+U v=0$ is disconjugate on $[a, \infty)$ (see the proof of Theorem 1). Therefore, by our induction hypothesis, there exists a solution $\tilde{\phi}(t)$ satisfying $\tilde{\phi}^{\prime \prime}+U \tilde{\phi}=0, \tilde{\phi}(a)=0$, and $\tilde{\phi}(t)>0$ for $t>a$. The vector $\psi(t)=$ $\operatorname{col}(\phi(t), \tilde{\phi}(t))$ is a solution of (8), which vanishes at $a$ and has strictly positive components on $(a, \infty)$. Hence $D^{T} \psi$ is the desired solution of (1).

The following example shows that the preceding theorem is false for the nonselfadjoint case.

Example 2. Let

$$
A(t)=\left[\begin{array}{ll}
1 / 4 t^{2} & 1 \\
0 & 1 / 4 t^{2}
\end{array}\right], \quad t \geqslant 1
$$




\section{It follows that}

$$
x^{\prime \prime}+A(t) x=0
$$

is disconjugate on $[1, \infty)$. For, if $x=\operatorname{col}(u, v)$ is a solution, then $u$ and $v$ satisfy the equations

$$
u^{\prime \prime}+\frac{1}{4 t^{2}} u+v=0
$$

and

$$
v^{\prime \prime}+\frac{1}{4 t^{2}} v=0
$$

The general solution of $(10)$ is $v(t)=c_{1} t^{1 / 2}+c_{2} t^{1 / 2} \ln t$. Therefore, if we assume the existence of two distinct numbers $a$ and $b ; a, b \geqslant 1$, satisfying $x(a)=x(b)=0$. then we must have $v(t) \equiv 0$. Now, a repetition of this argument shows that $u(t) \equiv 0$. This shows that $(1)$ is disconjugate on $[1, \infty)$.

Next, we wish to show that (1) does not have any solution $y(t)$ such that $y(1)=0$ and $y(t)>0$ for all $t, t>1$. Suppose that such a solution $y(t)$ exists. If we let $y(t)=\operatorname{col}(u(t), v(t))$, then the preceding argument and the fact that $v(1)=0$ imply that $v(t)=c_{2} t^{1 / 2} \ln t, c_{2}>0$. Since $u^{\prime \prime}=-\left(\left(1 / 4 t^{2}\right) u+v\right)$, we have $u^{\prime \prime}(t)<0$ for all $t, t>1$. Therefore, we must have $u^{\prime}(t)>0$ for $t>1$. On the other hand.

$$
u^{\prime}(t)-u^{\prime}(1)=\int_{1}^{t} u^{\prime \prime}(s) d s<\int_{1}^{t}-v(s) d s=-c_{2} \int_{1}^{t} s^{1 / 2} \ln s d s .
$$

But this leads to a contradiction since $-c_{2} \int_{1} s^{1 / 2} \ln s d s \rightarrow-\infty$ as $t \rightarrow \infty$.

Question. Are the conjugate points of a number relative to the nonselfadjoint system (1) isolated? (This question was raised in [6] and is still open.)

\section{REFERENCES}

I. S. Ahmad and A. C. Lazer. On the components of extremal solutions of second order ststem. SIAM J Math. Anal. 8 (1977), 16-23.

2. An N-dimensional extension of the Sturm separation and comparison theorn an a class of nonselfadjont sistems. SIAM J. Math. Anal. 9(1978), 1137-1150.

3. Positite operators and Sturmian theory of nonselfadjoint second order sistems. Nonlincar Equations in Abstract Spaces, Academic Press. New York, 1978, pp. 25-42.

4. $\quad$ A nen generalizatin of the Sturm comparison theorem to selfadjoint sistem.s. Proc. Amer. Math. Soc. 68 (1978), 185-188.

5. On an extension of Sturm's comparison theorem to a class of nonselfadiont second order stistems. Nonlinear Anal. 4 (1980), 497-501.

6. S. Ahmad, On positivity of solutions and conjugate points of nonselfadjoint sistems. Bull. Acad. Polon. Sci. Math. Astronom. Phys. 27 (1979), $71-75$.

7. S. Ahmad and J. A. Salazar, Conjugate points and second order si:̈tems. J. Math. Anal. Appl. 84 (1981), 63-72.

8. W. A. Coppel. Discomjugacy, Lecture Notes in Math., vol. 220). Springer-Verlag. Berlin and New York. 1971

9. M. Morse, A generalization of the Sturm separation and comparison theorems in 1-space. Math. Ann. $103(193()) .72-9()$.

10. K. Schmitt and H. L. Smith, Positive solutions and comjugate points for sistems of differential equations. Nonlinear Anal. 2 (1978), 93-105.

II. J. C. F. Sturm. Memoire sur les équations differentielles linesares de second ordre. J. Math. Pures Appl. 1 (1836). 106-186.

Department of Mathematics. University of Miami, Coral. (jabilis. Fi.orid)a 33124 